Pacific

Journal of

Mathematics

GRADIENT AND HARNACK INEQUALITIES ON NONCOMPACT MANIFOLDS WITH BOUNDARY

FENG-YU WANG 


\title{
GRADIENT AND HARNACK INEQUALITIES ON NONCOMPACT MANIFOLDS WITH BOUNDARY
}

\author{
FENG-YU WANG
}

\begin{abstract}
By using the reflecting diffusion process and a conformal change of metric, a generalized maximum principle is established for (unbounded) time-space functions on a class of noncompact Riemannian manifolds with (nonconvex) boundary. As applications, Li-Yau-type gradient and Harnack inequalities are derived for the Neumann semigroup on a class of noncompact manifolds with (nonconvex) boundary. These generalize some previous ones obtained for the Neumann semigroup on compact manifolds with boundary. As a byproduct, the gradient inequality for the Neumann semigroup derived by Hsu on a compact manifold with boundary is confirmed on these noncompact manifolds.
\end{abstract}

\section{Introduction}

Suppose $M$ is a $d$-dimensional connected complete Riemannian manifold, and let $L=\Delta+Z$, where $Z$ is a $C^{1}$ vector field satisfying the curvature-dimension condition of Bakry and Émery [1984] given by

(1-1) $\Gamma_{2}(f, f):=\frac{1}{2} L|\nabla f|^{2}-\langle\nabla L f, \nabla f\rangle \geq \frac{(L f)^{2}}{m}-K|\nabla f|^{2} \quad$ for $f \in C^{\infty}(M)$

for some constants $K \geq 0$ and $m>d$. By [Qian 1998, page 138], this condition is equivalent to

$$
\text { Ric }-\nabla Z-\frac{Z \otimes Z}{m-d} \geq-K \text {. }
$$

When $Z=0$ and $M$ is either without boundary or compact and with a convex boundary $\partial M, \mathrm{Li}$ and Yau [1986] found a now-famous gradient estimate for the (Neumann) semigroup $P_{t}$ generated by $L$ :

$$
\left|\nabla \log P_{t} f\right|^{2}-\alpha \partial_{t} \log P_{t} f \leq \frac{d \alpha^{2}}{2 t}+\frac{d \alpha^{2} K}{4(\alpha-1)} \quad \text { for } t>0 \text { and } \alpha>1
$$

MSC2000: 58J35, 60J60.

Keywords: gradient estimate, Harnack inequality, generalized maxium principle.

Supported in part by National Natural Science Foundation of China, number 10721091, and the 973 Project. 
for all positive $f \in C_{b}(M)$. We note that in [Li and Yau 1986] the second term on the right side of (1-3) is $d \alpha^{2} K /(\sqrt{2}(\alpha-1))$, but $\sqrt{2}$ here can be replaced by 4 according to a refined calculation; see for example [Davies 1989].

As an application, (1-3) implies a parabolic Harnack inequality for $P_{t}$ :

$$
P_{t} f(x) \leq\left(\frac{t+s}{t}\right)^{d \alpha / 2}\left(P_{t+s} f(y)\right) \exp \left(\frac{\alpha \rho(x, y)^{2}}{4 s}+\frac{\alpha K d s}{4(\alpha-1)}\right)
$$

for $t>0$ and $x, y \in M$,

where $\alpha>1$ and $f \in C_{b}(M)$ is positive. From this Harnack inequality, one obtains Gaussian-type heat kernel bounds for $P_{t}$; see [Li and Yau 1986; Davies 1989].

The gradient estimate (1-3) has been extended and improved in several papers. See for example [Bakry and Qian 1999] for an improved version for $\alpha=1$ with $Z \neq 0$ and $\partial M=\varnothing$, and see [Wang 1997] for an extension to a compact manifold with nonconvex boundary. The aim of this paper is to investigate the gradient and Harnack inequalities for $P_{t}$ on noncompact manifolds with (nonconvex) boundary.

Recall that the key step of Li and Yau's argument for the gradient estimate (1-3) is to apply the maximum principle to the reference function

$$
G(t, x):=t\left(\left|\nabla \log P_{t} f\right|^{2}-\alpha \partial_{t} \log P_{t} f\right)(x) \text { for } t \in[0, T] \text { and } x \in M .
$$

When $M$ is compact without boundary, the maximum principle says that for any smooth function $G$ on $[0, T] \times M$ with $G(0, \cdot) \leq 0$ and $\sup G>0$, there exists a maximal point of $G$ at which $\nabla G=0, \partial_{t} G \geq 0$, and $\Delta G \leq 0$. When $M$ is compact with a convex boundary, the same assertion holds for the above specified function $G$, as observed in [Li and Yau 1986, proof of Theorem 1.1]. In [1997], J. Wang extended this maximum principle on a compact manifold with nonconvex boundary by taking

$$
G(t, x)=t\left(\phi\left|\nabla \log P_{t} f\right|^{2}-\alpha \partial_{t} \log P_{t} f\right)(x) \quad \text { for } t \in[0, T] \text { and } x \in M
$$

for a nice function $\phi$ compensating the concavity of the boundary.

As for a noncompact manifold without boundary, Li and Yau [1986] established the gradient estimate by applying the maximal principle to a sequence of functions with compact support that approximate the original function $G$. An alternative is to apply directly the following generalized maximum principle:

Lemma 1.1 [Yau 1975]. For any bounded smooth function $G$ on $[0, T] \times M$ with $G(0, \cdot) \leq 0$ and $\sup G>0$, there exists a sequence $\left\{\left(t_{n}, x_{n}\right)\right\}_{n \geq 1} \subset[0, T] \times M$ such that

(i) $0<G\left(t_{n}, x_{n}\right) \uparrow \sup G$ as $n \uparrow \infty$, and

(ii) for any $n \geq 1$,

$$
L G\left(t_{n}, x_{n}\right) \leq 1 / n, \quad\left|\nabla G\left(t_{n}, \cdot\right)\left(x_{n}\right)\right| \leq 1 / n, \quad \partial_{t} G\left(t_{n}, x_{n}\right) \geq 0 .
$$


To apply this generalized maximal principle for the gradient estimate, one has to first confirm the boundedness of $G(t, \cdot):=t\left(\left|\nabla \log P_{t} f\right|^{2}-\alpha \partial_{t} \log P_{t} f\right)$ on $[0, T] \times M$ for $T>0$.

Since the boundedness of this type of reference function is unknown when $M$ is noncompact with a nonconvex boundary, we shall establish a generalized maximum principle on a class of noncompact manifolds with boundary for not necessarily bounded functions. Applying this principle to a suitable reference function $G$, we derive the Li-Yau-type gradient and Harnack inequalities for Neumann semigroups. To establish such a maximum principle, we adopt a localization argument so that the classical maximum principle can be applied.

For $M$ noncompact without boundary, Li and Yau [1986] used such a localization argument to apply the maximal principle to functions with compact support; they then passed to the desired global estimate by taking a limit. To do this, they constructed cut-off functions using $\rho_{o}$, the Riemannian distance function to a fixed point $o \in M$. It turns out that this argument works also when $\partial M$ is convex; see Section 2.1. For the nonconvex case, we will use the conformal change of metric introduced in [Wang 2007] to make a nonconvex boundary convex; see Section 2.2.

Assumption A. The manifold $M$ is connected and complete with boundary $\partial M$ and such that either

(1) $\partial M$ is convex, or

(2) the second fundamental form of $\partial M$ is bounded, the sectional curvature of $M$ is bounded from above, and the injectivity radius $\mathrm{i}_{\partial M}$ of $\partial M$ is positive.

Recall that the Riemannian distance function $\rho_{\partial M}$ to the boundary is smooth on the set $\left\{\rho_{\partial M}<\mathrm{i}_{\partial M}\right\}$.

Let $N$ be the inward unit normal vector field on $\partial M$. The second fundamental form of $\partial M$ is

$$
\mathrm{II}(X, Y)=-\left\langle\nabla_{X} N, Y\right\rangle \quad \text { for } X, Y \in T \partial M .
$$

The boundary $\partial M$ is called convex if $I I \geq 0$. We are now ready to state our generalized maximal principle for possibly unbounded functions.

Theorem 1.2. Let $M$ satisfy $A$, and let $L$ satisfy (1-2). Let $T>0$, and let $G$ be a smooth function on $[0, T] \times M$ such that $\left.N G\right|_{\partial M} \geq 0, G(0, \cdot) \leq 0$ and $\sup G>0$. Then for any $\varepsilon>0$, there exists a sequence $\left\{\left(t_{n}, x_{n}\right)\right\}_{n \geq 1} \subset(0, T] \times M$ such that Lemma 1.1(i) holds and for any $n \geq 1$

$$
\begin{aligned}
& L G\left(t_{n}, x_{n}\right) \leq \frac{G\left(t_{n}, x_{n}\right)^{1+\varepsilon}}{n}, \quad\left|\nabla G\left(t_{n}, x_{n}\right)\right| \leq \frac{G\left(t_{n}, x_{n}\right)^{1+\varepsilon}}{n}, \\
& \partial_{t} G\left(t_{n}, x_{n}\right) \geq 0 .
\end{aligned}
$$


Applying Theorem 1.2 to a proper choice of function $G$, we will derive the Li-Yau-type gradient estimate (1-5). We shall prove that the reflecting diffusion process $X_{t}$ generated by $L$ on $M$ is non explosive, so that the corresponding Neumann semigroup $P_{t}$ can be formulated as

$$
P_{t} f(x)=\mathrm{E}^{x} f\left(X_{t}\right) \quad \text { for } t \geq 0, x \in M \text {, and } f \in C_{b}(M),
$$

where $\mathrm{E}^{x}$ is the expectation taken for $X_{0}=x$.

Theorem 1.3. Let $M$ satisfy $A$, and suppose $L$ satisfies (1-2) with $\|Z\|_{\infty}<\infty$. Then the reflecting $L$-diffusion process on $M$ is nonexplosive and the corresponding Neumann semigroup $P_{t}$ satisfies these assertions:

(i) If $\partial M$ is convex, then (1-3) holds with $m$ in place of $d$.

(ii) If $\partial M$ is nonconvex with $\mathrm{II} \geq-\sigma$ for some $\sigma>0$, then for any bounded $\phi \in C^{\infty}(M)$ with $\phi \geq 1$ and $\left.N \log \phi\right|_{\partial M} \geq 2 \sigma$, the gradient inequality

$$
\left|\nabla \log P_{t} f\right|^{2}-\alpha \partial_{t} \log P_{t} f \leq \frac{m(1+\varepsilon) \alpha^{2}}{2(1-\varepsilon) t}+\frac{m \alpha^{2} K(\phi, \varepsilon, \alpha)}{4\left(\alpha-\|\phi\|_{\infty}\right)}
$$

holds for all positive $f \in C_{b}(M), \alpha>\|\phi\|_{\infty}, t>0, \varepsilon \in(0,1)$ and $K(\phi, \varepsilon, \alpha):=$

$$
\frac{1+\varepsilon}{1-\varepsilon}\left(K+\frac{1}{\varepsilon}\|\nabla \log \phi\|_{\infty}^{2}+\frac{1}{2} \sup \left(-\phi^{-1} L \phi\right)+\frac{m \alpha^{2}\|\nabla \log \phi\|_{\infty}^{2}(1+\varepsilon)}{8\left(\alpha-\|\phi\|_{\infty}\right)^{2} \varepsilon(1-\varepsilon)}\right) .
$$

We emphasize that the results in Theorem 1.3 are new for noncompact manifolds with boundary. When $M$ is compact with a convex boundary, the first assertion was proved in [Li and Yau 1986] by using the classical maximum principle on compact manifolds, while when $M$ is compact with a nonconvex boundary, an inequality similar to (1-5) was proved in [Wang 1997] by using the "interior rolling $R$-ball" condition.

These two theorems will be proved in Sections 2 and 3. By a standard argument due to Li and Yau [1986], the gradient estimate (1-5) implies a Harnack inequality. Let $\rho(x, y)$ be the Riemannian distance between $x, y \in M$, that is, the infimum of the length of all smooth curves in $M$ that link $x$ and $y$.

Corollary 1.4. In the situation of Theorem 1.3 the Neumann semigroup $P_{t}$ satisfies

$$
\begin{aligned}
& P_{t} f(x) \leq \\
& \quad\left(\frac{t+s}{t}\right)^{m(1+\varepsilon) \alpha / 2(1-\varepsilon)}\left(P_{t+s} f(y)\right) \exp \left(\frac{\alpha \rho(x, y)^{2}}{4 s}+\frac{\alpha m K(\phi, \varepsilon, \alpha) s}{4\left(\alpha-\|\phi\|_{\infty}\right)}\right)
\end{aligned}
$$

for all positive $f \in C_{b}(M), t, \varepsilon \in(0,1), \alpha>\|\phi\|_{\infty}$ and $x, y \in M$. In particular, if $\partial M$ is convex, then (1-4) holds with $m$ in place of $d$ and for all $\alpha>1$. 
To derive explicit inequalities for the nonconvex case, we shall take a specific choice of $\phi$ as in [Wang 2007]. Let $\mathrm{i}_{\partial M}$ be the injectivity radius of $\partial M$, and let $\rho_{\partial M}$ be the Riemannian distance to the boundary. We shall take $\phi=\varphi \circ \rho_{\partial M}$ for a nice reference function $\varphi$ on $[0, \infty)$. More precisely, let the sectional curvature satisfy $\operatorname{Sect}_{M} \leq k$ and $-\sigma \leq \mathrm{II} \leq \gamma$ for some $k, \sigma, \gamma>0$. Let

$$
h(s)=\cos (\sqrt{k} s)-(\gamma / \sqrt{k}) \sin (\sqrt{k} s) \text { for } s \geq 0 .
$$

Then $h$ is the unique solution to the differential equation $h^{\prime \prime}+k h=0$ with boundary conditions $h(0)=1$ and $h^{\prime}(0)=-\gamma$. By the Laplacian comparison theorem for $\rho_{\partial M}$ (see [Kasue 1984, Theorem 0.3] or [Wang 2007]),

$$
\Delta \rho_{\partial M} \geq \frac{(d-1) h^{\prime}}{h}\left(\rho_{\partial M}\right) \quad \text { and } \quad \rho_{\partial M}<\mathrm{i}_{\partial M} \wedge h^{(-1)}(0),
$$

where $h^{(-1)}(0)=(1 / \sqrt{k}) \arcsin \left(\sqrt{k} / \sqrt{k+\gamma^{2}}\right)$ is the first zero point of $h$. Fix a positive number $r_{0} \leq \mathrm{i}_{\partial M} \wedge h^{(-1)}(0)$, and let

$$
\begin{aligned}
\delta & =\frac{2 \sigma\left(1-h\left(r_{0}\right)\right)^{d-1}}{\int_{0}^{r_{0}}\left(h(s)-h\left(r_{0}\right)\right)^{d-1} \mathrm{~d} s}, \\
\varphi(r) & =1+\delta \int_{0}^{r}\left(h(s)-h\left(r_{0}\right)^{1-d} \mathrm{~d} s \int_{s \wedge r_{0}}^{r_{0}}\left(h(u)-h\left(r_{0}\right)\right)^{d-1} \mathrm{~d} u .\right.
\end{aligned}
$$

It is easy to see that $\varphi \circ \rho_{\partial M}$ is differentiable with a Lipschitzian gradient. By a simple approximation argument, we may apply Theorem 1.3 and Corollary 1.4 to $\phi=\varphi \circ \rho_{\partial M}$; see [Wang 2007, page 1436].

Obviously, (1-7) and $N=\nabla \rho_{\partial M}$ imply

$$
\Delta \varphi \circ \rho_{\partial M} \geq-\delta \quad \text { and }\left.\quad N \log \varphi \circ \rho_{\partial M}\right|_{\partial M}=\varphi^{\prime}(0) / \varphi(0)=2 \sigma .
$$

Moreover, by [Wang 2007, (20)] we have

$$
\delta \leq 2 \sigma d r_{0}^{-1} \text { and } \varphi\left(r_{0}\right) \leq 1+\sigma d r_{0} .
$$

Thus, for $\phi:=\varphi \circ \rho_{\partial M}$ we have

$$
\begin{aligned}
-\phi^{-1} L \phi & \leq 2 \sigma d r_{0}^{-1}+2 \sigma\|Z\|_{\infty}, \quad\|\nabla \log \phi\|_{\infty}^{2} \leq 4 \sigma^{2}, \\
\|\phi\|_{\infty} & \leq \varphi\left(r_{0}\right) \leq 1+\sigma d r_{0} .
\end{aligned}
$$

Combining these with Theorem 1.3 and Corollary 1.4, we obtain these explicit inequalities on a class of nonconvex and noncompact manifolds:

Corollary 1.5. Let $\mathrm{i}_{\partial M}>0$, and suppose $\gamma \geq \mathrm{II} \geq-\sigma$ and Sect $_{M} \leq k$ for some $\gamma, \sigma, k>0$. If (1-2) holds and $\|Z\|_{\infty}<\infty$, then for any positive number

$$
r_{0} \leq \min \left\{\mathrm{i}_{\partial M},(1 / \sqrt{k}) \arcsin \left(\sqrt{k} / \sqrt{k+\gamma^{2}}\right)\right\},
$$


the inequalities

$$
\left|\nabla \log P_{t} f\right|^{2}-\alpha \partial_{t} \log P_{t} f \leq \frac{m(1+\varepsilon) \alpha^{2}}{2(1-\varepsilon) t}+\frac{m \alpha^{2} K_{\varepsilon}}{4\left(\alpha-1-\sigma d r_{0}\right)}
$$

and

$$
\begin{array}{r}
P_{t} f(x) \leq\left(\frac{t+s}{t}\right)^{m(1+\varepsilon) \alpha / 2(1-\varepsilon)}\left(P_{t+s} f(y)\right) \exp \left(\frac{\alpha \rho(x, y)^{2}}{4 s}+\frac{m \alpha K_{\varepsilon} s}{4\left(\alpha-1-\sigma d r_{0}\right)}\right) \\
\quad \text { for } x, y \in M
\end{array}
$$

hold for all positive $f \in C_{b}(M), t>0, \varepsilon \in(0,1), \alpha>1+\sigma d r_{0}$, and

$$
K_{\varepsilon}=\frac{1+\varepsilon}{1-\varepsilon}\left(K+\frac{4 \sigma^{2}}{\varepsilon}+\frac{\sigma d}{r_{0}}+\sigma\|Z\|_{\infty}+\frac{m \alpha^{2} \sigma^{2}(1+\varepsilon)}{2\left(\alpha-1-\sigma d r_{0}\right)^{2} \varepsilon(1-\varepsilon)}\right) .
$$

Combining our gradient estimate with an approximation and a probabilistic argument, we can derive the gradient estimate (1-9) for a class of noncompact manifolds:

Theorem 1.6. Let $M$ satisfy $A$, and let $L$ satisfy (1-2) with $\|Z\|_{\infty}<\infty$. Let $\kappa_{1}$ and $\kappa_{2}$ be positive elements of $C_{b}(M)$ such that

$$
\text { Ric }-\nabla Z \geq-\kappa_{1} \quad \text { and } \quad \mathrm{II} \geq-\kappa_{2}
$$

hold on $M$ and $\partial M$, respectively. Then

$$
\left|\nabla P_{t} f\right|(x) \leq \mathrm{E}^{x}\left(|\nabla f|\left(X_{t}\right) \exp \left(\int_{0}^{t} \kappa_{1}\left(X_{s}\right) \mathrm{d} s+\int_{0}^{t} \kappa_{2}\left(X_{s}\right) \mathrm{d} l_{s}\right)\right)
$$

holds for all $f \in C_{b}^{1}(M), t>0$, and $x \in M$.

Inequality (1-9) was first derived by Hsu [2002] on a compact manifold with boundary. In [2002, Theorem 3.7], Hsu applied the Itô formula to $F\left(U_{t}, T-\right.$ $t):=U_{t}^{-1} \nabla P_{T-t} f\left(X_{t}\right)$, where $U_{t}$ is the horizontal lift of $X_{t}$ on the frame bundle $O(M)$. Since $M$ is compact, the (local) martingale part of this process is a real martingale (it may not be for noncompact $M$ ). Then the desired gradient estimate followed immediately from [2002, Corollary 3.6]. In Section 4, we will prove the boundedness of $\nabla P_{(\cdot)} f$ on $[0, T] \times M$ for any $T>0$ and $f \in C_{b}^{1}(M)$, which leads to a simple proof of (1-9) for a class of noncompact manifolds.

\section{Proof of Theorem 1.2}

We consider the convex case and pass to the nonconvex case using the conformal change of metric constructed in [Wang 2007]. Without loss of generality, we may assume that $\sup G:=\sup _{[0, T] \times M}>1$. (Otherwise, we simply replace $G$ by $m G$ for a sufficiently large $m>0$.) 
2.1. Convex $\boldsymbol{\partial}$ M. Fix $o \in M$, and let $\rho_{o}$ be the Riemannian distance to the point $o$. Since $\partial M$ is convex, there exists a minimal geodesic in $M$ of length $\rho(x, y)$ that links any $x$ and $y$ in $M$; see for example [Wang 2005a, Proposition 2.1.5]. So, by (1-2) and a comparison theorem (see [Qian 1998])

$$
L \rho_{o} \leq \sqrt{K(m-1)} \operatorname{coth}\left(\sqrt{K /(m-1)} \rho_{o}\right)
$$

holds outside $\{o\} \cup \operatorname{cut}(o)$, where $\operatorname{cut}(o)$ is the cut locus of $o$. In the sequel, we will set $L \rho_{o}=0$ on $\operatorname{cut}(o)$ so that this implies

$$
L \sqrt{1+\rho_{o}^{2}} \leq c_{1} \quad \text { on } M
$$

for some constant $c_{1}>0$.

Let $h \in C_{0}^{\infty}([0, \infty))$ be decreasing such that

$$
h(r)= \begin{cases}1 & \text { if } r \leq 1 \\ \exp \left(-(3-r)^{-1}\right) & \text { if } r \in[2,3) \\ 0 & \text { if } r \geq 3\end{cases}
$$

Obviously, for any $\varepsilon>0$ we have

$$
\sup _{[0, \infty)}\left\{\left|h^{\varepsilon-1} h^{\prime \prime}\right|+\left|h^{\varepsilon-1} h^{\prime}\right|\right\}<\infty .
$$

Let $W=\sqrt{1+\rho_{o}^{2}}$, and take $\varphi_{n}=h(W / n)$ for $n \geq 1$. Then

$$
\left\{\varphi_{n}=1\right\} \uparrow M \quad \text { as } n \uparrow \infty .
$$

So, according to (2-1) and (2-2),

$$
\begin{aligned}
\left|\nabla \log \varphi_{n}\right| & \leq \frac{c}{n \varphi_{n}^{\varepsilon}} \\
\varphi_{n}^{-1} L \varphi_{n} & =\frac{h^{\prime}(W / n)}{n h(W / n)} L W+\frac{h^{\prime \prime}(W / n)}{n^{2} h(W / n)}|\nabla W|^{2} \geq-\frac{c}{n \varphi_{n}^{\varepsilon}}
\end{aligned}
$$

holds for some constant $c>0$ and all $n \geq 1$.

Let $G_{n}(t, x)=\varphi_{n}(x) G(t, x)$ for $t \in[0, T]$ and $x \in M$. Since $G_{n}$ is continuous with compact support, there exists $\left(t_{n}, x_{n}\right) \in[0, T] \times M$ such that

$$
G_{n}\left(t_{n}, x_{n}\right)=\max _{[0, T] \times M} G_{n} .
$$

By (2-3) and that $\sup G>1$, we have $\lim _{n \rightarrow \infty} G\left(t_{n}, x_{n}\right)=\sup G>1$. By renumbering from a sufficient large $n_{0}$, we may assume that $G_{n}\left(t_{n}, x_{n}\right)$ is greater than 1 and is increasing in $n$. In particular, Lemma 1.1(i) holds and

$$
\varphi_{n}\left(x_{n}\right) \geq 1 / G\left(t_{n}, x_{n}\right) \quad \text { for } n \geq 1 .
$$


Moreover, since $G_{n}(0, \cdot) \leq 0$, we have $t_{n}>0$ and $\partial_{t} G\left(t_{n}, x_{n}\right) \geq 0$ for $n \geq 1$. Thus, it remains to confirm that

$$
\begin{array}{rlrl}
\left|\nabla G\left(t_{n}, x_{n}\right)\right| & \leq c G\left(t_{n}, x_{n}\right)^{1+\varepsilon} / n & & \text { and } \\
L G\left(t_{n}, x_{n}\right) \leq c G\left(t_{n}, x_{n}\right)^{1+\varepsilon} / n & & \text { for } n \geq 1
\end{array}
$$

for some constant $c>0$. Indeed, by using a subsequence $\left\{\left(t_{m n}, x_{m n}\right)\right\}_{n \geq 1}$ for $m \geq c$ to replace $\left\{\left(t_{n}, x_{n}\right)\right\}_{n \geq 1}$, one may reduce (2-6) with some $c>0$ to that with $c=1$.

Since $x_{n}$ is the maximal point of $G_{n}$, we have $\nabla G_{n}\left(t_{n}, x_{n}\right)=0$ if $x_{n} \in M \backslash \partial M$. If $x_{n} \in \partial M$, we have $N G_{n}\left(t_{n}, x_{n}\right) \leq 0$. Recall that $N G\left(t_{n}, \cdot\right) \geq 0$ and $G\left(t_{n}, x_{n}\right)>0$. Then, noting that $N \rho_{0} \leq 0$ together with $h^{\prime} \leq 0$ implies $N \varphi_{n} \geq 0$, we conclude that $N G_{n}\left(t_{n}, x_{n}\right) \geq 0$. Hence, $N G_{n}\left(t_{n}, x_{n}\right)=0$. Moreover, since $x_{n}$ is the maximal point of $G_{n}\left(t_{n}, \cdot\right)$ on the closed manifold $\partial M$, we have $U G_{n}\left(t_{n}, x_{n}\right)=0$ for all $U \in T \partial M$. Therefore, $\nabla G_{n}\left(t_{n}, x_{n}\right)=0$ also holds for $x_{n} \in \partial M$. Combining this with (2-4) and (2-5), we obtain

$$
\left|\nabla G\left(t_{n}, x_{n}\right)\right| \leq \frac{G\left(t_{n}, x_{n}\right)}{\varphi_{n}\left(x_{n}\right)}\left|\nabla \varphi_{n}\right| \leq \frac{c G\left(t_{n}, x_{n}\right)^{1+\varepsilon}}{n},
$$

which proves the first inequality in (2-6).

Finally, by (2-4), the inequality

$$
\varphi_{n} L_{n} G+G L_{n} \varphi_{n}+2\left\langle\nabla G, \nabla \varphi_{n}\right\rangle \geq \varphi_{n} L_{n} G-\frac{c \varphi_{n}^{1-\varepsilon}}{n} G-\frac{2 c \varphi_{n}^{1-\varepsilon}}{n}|\nabla G|=: \Phi
$$

holds on $\left\{G_{n}>0\right\} \backslash \operatorname{cut}(o)$. By Lemma 2.1 below we obtain at the point $\left(t_{n}, x_{n}\right)$ that

$$
L G \leq \frac{c}{n \varphi_{n}^{\varepsilon}} G+\frac{2 c}{n \varphi_{n}}|\nabla G| .
$$

Combining this with (2-5) and the first inequality in (2-6), we get

$$
L G\left(t_{n}, x_{n}\right) \leq \frac{c}{n} G^{1+2 \varepsilon}\left(t_{n}, x_{n}\right)
$$

for some constant $c>0$ and all $n \geq 1$. Since $\varepsilon>0$ is arbitrary, we may replace $\varepsilon$ by $\varepsilon / 2$ (recall that $G\left(t_{n}, x_{n}\right) \geq 1$ ). This proves the second inequality in (2-6).

Lemma 2.1. The reflecting $L$-diffusion process is nonexplosive, and for any $\Phi$ in $C_{b}(M)$ such that

$$
\Phi \leq L G_{n}=G L \varphi_{n}+\varphi_{n} L G+2\left\langle\nabla \varphi_{n}, \nabla G\right\rangle \text { on }\left\{G_{n}>0\right\} \backslash \operatorname{cut}(o),
$$

we have $\Phi\left(t_{n}, x_{n}\right) \leq 0$ for all $n \geq 1$.

Proof. Let $X_{t}$ be the reflecting $L$-diffusion process generated by $L$, and let $U_{t}$ be its horizontal lift on the frame bundle $O(M)$. By the Itô formula for $\rho_{o}\left(X_{t}\right)$ 
found by Kendall [1987] for $\partial M=\varnothing$ and by the fact that $\left.N \rho_{o}\right|_{\partial M} \leq 0$ when $\partial M$ is nonempty but convex, we have

$$
\mathrm{d} \rho_{o}\left(X_{t}\right)=\sqrt{2}\left\langle\nabla \rho_{o}\left(X_{t}\right), U_{t} \mathrm{~d} B_{t}\right\rangle+L \rho_{o}\left(X_{t}\right) \mathrm{d} t-\mathrm{d} l_{t}+\mathrm{d} l_{t}^{\prime},
$$

where $B_{t}$ is the $d$-dimensional Brownian motion, where $L \rho_{o}$ is taken to be zero on $\{o\} \cup \operatorname{cut}(o)$, and where $l_{t}$ and $l_{t}^{\prime}$ are two increasing processes such that $l_{t}^{\prime}$ increases only when $X_{t}=o$, while $l_{t}$ increases only when $X_{t} \in \operatorname{cut}(o) \cup \partial M$ (note that $l_{t}^{\prime}=0$ for $d \geq 2$ ). Combining this with (2-1) we obtain

$$
\mathrm{d} \sqrt{1+\rho_{o}^{2}\left(X_{t}\right)} \leq \mathrm{d} M_{t}+L \sqrt{1+\rho_{o}^{2}\left(X_{t}\right)} \mathrm{d} t \leq \mathrm{d} M_{t}+c_{1} \mathrm{~d} t
$$

for some martingale $M_{t}$. This implies immediately that $X_{t}$ does not explode.

Now, let us take $X_{0}=x_{n}$. Since $h^{\prime} \leq 0$, it follows from (2-7) that

$$
\mathrm{d} \varphi_{n}\left(X_{t}\right) \geq \sqrt{2}\left\langle\nabla \varphi_{n}\left(X_{t}\right), U_{t} \mathrm{~d} B_{t}\right\rangle+L \varphi_{n}\left(X_{t}\right) \mathrm{d} t,
$$

where we set $L \varphi_{n}=0$ on $\operatorname{cut}(o)$ as above.

On the other hand, since $N G\left(t_{n}, \cdot\right) \geq 0$, we may apply the Itô to $G\left(t_{n}, X_{t}\right)$ to obtain

$$
\mathrm{d} G\left(t_{n}, X_{t}\right) \geq \sqrt{2}\left\langle\nabla G\left(t_{n}, X_{t}\right), U_{t} \mathrm{~d} B_{t}\right\rangle+L G\left(t_{n}, X_{t}\right) \mathrm{d} t .
$$

Because $G_{n}\left(t_{n}, x_{n}\right)>0$, there exists an $r>0$ such that $G_{n}>0$ on $B\left(x_{n}, r\right)$, the geodesic ball in $M$ centered at $x_{n}$ with radius $r$. Let

$$
\tau=\inf \left\{t \geq 0: X_{t} \notin B\left(x_{n}, r\right)\right\} .
$$

Then (2-8) and (2-9) imply

$$
\mathrm{d} G_{n}\left(t_{n}, X_{t}\right) \geq \mathrm{d} M_{t}+L G_{n}\left(t_{n}, \cdot\right)\left(X_{t}\right) \mathrm{d} t \geq \mathrm{d} M_{t}+\Phi\left(t_{n}, X_{t}\right) \mathrm{d} t \quad \text { for } t \leq \tau
$$

for some martingale $M_{t}$. Since $G_{n}\left(t_{n}, X_{t}\right) \leq G_{n}\left(t_{n}, x_{n}\right)$ and $X_{0}=x_{n}$, this implies that

$$
0 \geq \mathrm{E} G_{n}\left(t_{n}, X_{t \wedge \tau}\right)-G_{n}\left(t_{n}, x_{n}\right) \geq \mathrm{E} \int_{0}^{t \wedge \tau} \Phi\left(t_{n}, X_{s}\right) \mathrm{d} s .
$$

Therefore, the continuity of $\Phi$ implies that

$$
\Phi\left(t_{n}, x_{n}\right)=\lim _{t \rightarrow 0} \frac{1}{\mathrm{E}(t \wedge \tau)} \mathrm{E} \int_{0}^{t \wedge \tau} \Phi\left(t_{n}, X_{s}\right) \mathrm{d} s \leq 0 .
$$

2.2. Nonconvex $\boldsymbol{\partial} \boldsymbol{M}$. Under our assumptions on $M$, there exists a constant $R>1$ and a function $\phi \in C^{\infty}(M)$ such that

$$
1 \leq \phi \leq R, \quad|\nabla \phi| \leq R,\left.\quad N \log \phi\right|_{\partial M} \geq \sigma .
$$

By [Wang 2007, Lemma 2.1], the boundary $\partial M$ is convex under the new metric $\langle\cdot, \cdot\rangle^{\prime}:=\phi^{-2}\langle\cdot, \cdot\rangle$. Let $L^{\prime}=\phi^{2} L$. By [Wang 2007, Equation (9)], the vector 
$U^{\prime}:=\phi U$ is unit under the new metric for any unit vector $U \in T M$, and the corresponding Ricci curvature satisfies

$$
\begin{aligned}
\operatorname{Ric}^{\prime}\left(U^{\prime}, U^{\prime}\right) \geq \phi^{2} \operatorname{Ric}(U, U)+\phi \Delta \phi & -(d-3)|\nabla \phi|^{2} \\
& -2(U \phi)^{2}+(d-2) \phi \operatorname{Hess}_{\phi}(U, U) .
\end{aligned}
$$

Let $\Delta^{\prime}$ be the Laplacian induced by the new metric. By [Wang 2007, Lemma 2.2], we have

$$
L^{\prime}:=\phi^{2} L=\Delta^{\prime}+(d-2) \phi \nabla \phi+\phi^{2} Z=: \Delta^{\prime}+Z^{\prime} .
$$

Noting that

$\nabla_{X}^{\prime} Y=\nabla_{X} Y-\langle X, \nabla \log \phi\rangle Y-\langle Y, \nabla \log \phi\rangle X+\langle X, Y\rangle \nabla \log \phi \quad$ for $X, Y \in T M$, we have

$$
\begin{aligned}
\left\langle\nabla_{U^{\prime}} Z^{\prime}, U^{\prime}\right\rangle^{\prime} & =\left\langle\nabla_{U} Z^{\prime}, U\right\rangle-\left\langle Z^{\prime}, \nabla \log \phi\right\rangle \\
= & \phi^{2}\left\langle\nabla_{U} Z, U\right\rangle+\left(U \phi^{2}\right)\langle Z, U\rangle+(d-2)(U \phi)^{2} \\
& +(d-2) \phi \operatorname{Hess}_{\phi}(U, U)-\left\langle Z^{\prime}, \nabla \log \phi\right\rangle .
\end{aligned}
$$

Combining this with (2-10) and the properties of $\phi$ mentioned above, we find a constant $c_{1}>0$ such that

(2-11) $\operatorname{Ric}^{\prime}\left(U,{ }^{\prime} U^{\prime}\right)-\left\langle\nabla_{U^{\prime}}^{\prime} Z^{\prime}, U^{\prime}\right\rangle^{\prime} \geq \phi^{2}(\operatorname{Ric}-\nabla Z)(U, U)-c_{1} \quad$ for $|U|=1$.

Moreover, since

$$
\begin{aligned}
\left(Z^{\prime} \otimes^{\prime} Z^{\prime}\right)\left(U^{\prime}, U^{\prime}\right):=\left(\left\langle Z^{\prime}, U^{\prime}\right\rangle^{\prime}\right)^{2} & =\phi^{-2}\left\langle Z^{\prime}, U\right\rangle^{2} \\
& \leq 2(d-2)^{2}\langle\nabla \phi, U\rangle^{2}+2 \phi^{2}\langle Z, U\rangle^{2} \\
& \leq 2(d-2)^{2} R^{2}+2 \phi^{2}(Z \otimes Z)(U, U),
\end{aligned}
$$

it follows from (1-2) and (2-11) that

$$
\operatorname{Ric}^{\prime}-\nabla^{\prime} Z^{\prime}-\frac{Z^{\prime} \otimes^{\prime} Z^{\prime}}{2(m-d)} \geq-\phi^{2} K-c_{2} \geq-K^{\prime}
$$

holds for the metric $\langle\cdot, \cdot\rangle^{\prime}$ and some constants $c_{2}, K^{\prime}>0$. Therefore, we may apply Lemma 2.1 to $L^{\prime}$ on the convex Riemannian manifold $\left(M,\langle\cdot, \cdot\rangle^{\prime}\right)$ to conclude that the desired sequence $\left\{\left(t_{n}, x_{n}\right)\right\}$ exists.

\section{Proofs of Theorem 1.3 and Corollary 1.4}

Proof of Theorem 1.3. When $\partial M$ is convex, Lemma 2.1 ensures that $X_{t}$ does not explode. If $\partial M$ is nonconvex, this can be confirmed by reparametrizing the time of the process. More precisely, let $X_{t}^{\prime}$ be the reflecting diffusion process on $M$ generated by $L^{\prime}:=\phi^{2} L$ constructed in Section 2.2. Since $L^{\prime}=\Delta^{\prime}+Z^{\prime}$ 
satisfies (1-2) for some $K>0$ on the convex manifold $\left(M,\langle\cdot, \cdot\rangle^{\prime}\right)$, the process $X_{t}^{\prime}$ generated by $L^{\prime}$ is nonexplosive by Lemma 2.1. Since $X_{t}=X_{\xi^{-1}(t)}^{\prime}$, where $\xi^{-1}$ is the inverse of

$$
t \mapsto \xi(t)=\int_{0}^{t} \phi^{2}\left(X_{s}^{\prime}\right) \mathrm{d} s,
$$

we have $t\|\phi\|_{\infty}^{-2} \leq \xi^{-1}(t) \leq t$, and the process $X_{t}$ is nonexplosive as well.

Let $f \in C_{b}^{1}(M)$ be strictly positive, and let $u(t, x)=\log P_{t} f(x)$. For a fixed number $T>0$, we will apply Theorem 1.2 to the reference function

$$
G(t, x)=t\left\{\phi(x)|\nabla u|^{2}(t, x)-\alpha u_{t}(t, x)\right\} \quad \text { for } t \in[0, T] \text { and } x \in M .
$$

Note that II $\geq-\sigma$ and $N \log \phi \geq 2 \sigma$ imply

$$
\begin{aligned}
N \phi & \geq 2 \sigma \phi, \\
N\left|\nabla P_{t} f\right|^{2} & =2 \operatorname{Hess}_{P_{t} f}\left(N, \nabla P_{t} f\right)=2 \Pi\left(\nabla P_{t} f, \nabla P_{t} f\right) \geq-2 \sigma\left|\nabla P_{t} f\right|^{2} .
\end{aligned}
$$

Since $P_{t} f$ and hence $u_{t}$ satisfy the Neumann boundary condition, this implies that $N G=t\left\{(N \phi)|\nabla u|^{2}+\frac{\phi}{\left(P_{t} f\right)^{2}} N\left|\nabla P_{t} f\right|^{2}\right\} \geq t\left\{2 \sigma \phi|\nabla u|^{2}-2 \sigma \phi|\nabla u|^{2}\right\}=0$ on $\partial M$.

According to [Ledoux 2000, (1.14)], inequality (1-2) implies

$$
L|\nabla u|^{2}-2\langle\nabla L u, \nabla u\rangle \geq-2 K|\nabla u|^{2}+\frac{\left.\left.|\nabla| \nabla u\right|^{2}\right|^{2}}{2|\nabla u|^{2}} .
$$

By multiplying this inequality by $\varepsilon$ and $(1-1)$ by $2(1-\varepsilon)$ and by combining the results, we obtain

$$
L|\nabla u|^{2} \geq 2\langle\nabla L u, \nabla u\rangle-2 K|\nabla u|^{2}+\frac{2(1-\varepsilon)(L u)^{2}}{m}+\frac{\left.\left.\varepsilon|\nabla| \nabla u\right|^{2}\right|^{2}}{2|\nabla u|^{2}} .
$$

It is also easy to check that $L u=u_{t}-|\nabla u|^{2}$ and $\partial_{t}|\nabla u|^{2}=2\left\langle\nabla u, \nabla u_{t}\right\rangle$. Then we arrive at

$$
\begin{aligned}
& \left(L-\partial_{t}\right)|\nabla u|^{2} \\
& \quad \geq \frac{2(1-\varepsilon)}{m}\left(|\nabla u|^{2}-u_{t}\right)^{2}+\frac{\left.\left.\varepsilon|\nabla| \nabla u\right|^{2}\right|^{2}}{2|\nabla u|^{2}}-2\left\langle\nabla u, \nabla|\nabla u|^{2}\right\rangle-2 K|\nabla u|^{2} .
\end{aligned}
$$

On the other hand,

$$
\begin{aligned}
-\alpha\left(L-\partial_{t}\right) u_{t} & =2 \alpha\left\langle\nabla u, \nabla u_{t}\right\rangle=2\left\langle\nabla u, \nabla\left(\phi|\nabla u|^{2}-t^{-1} G\right)\right\rangle \\
& =2 \phi\left\langle\nabla u, \nabla|\nabla u|^{2}\right\rangle+2|\nabla u|^{2}\langle\nabla u, \nabla \phi\rangle-2 t^{-1}\langle\nabla u, \nabla G\rangle .
\end{aligned}
$$


Combining this with (3-2), we obtain

$$
\begin{aligned}
\left(L-\partial_{t}\right) G=-\frac{G}{t}+ & t\left(\phi\left(L-\partial_{t}\right)|\nabla u|^{2}+|\nabla u|^{2} L \phi+2\left\langle\nabla \phi, \nabla|\nabla u|^{2}\right\rangle\right) \\
& \quad+t\left(2 \phi\left\langle\nabla u, \nabla|\nabla u|^{2}\right\rangle+2|\nabla u|^{2}\langle\nabla u, \nabla \phi\rangle-2 t^{-1}\langle\nabla u, \nabla G\rangle\right) \\
\geq-\frac{G}{t}+ & \frac{2(1-\varepsilon) \phi t}{m}\left(|\nabla u|^{2}-u_{t}\right)^{2}+\frac{\left.\left.\varepsilon \phi t|\nabla| \nabla u\right|^{2}\right|^{2}}{2|\nabla u|^{2}}-2 K \phi t|\nabla u|^{2} \\
& \quad 2|\nabla u| \cdot|\nabla G|-2 t|\nabla u|^{3}|\nabla \phi|-\left.\left.2 t|\nabla \phi| \cdot|\nabla| \nabla u\right|^{2}|+t| \nabla u\right|^{2} L \phi .
\end{aligned}
$$

Noting that

$$
\frac{\left.\left.\varepsilon \phi t|\nabla| \nabla u\right|^{2}\right|^{2}}{2|\nabla u|^{2}}-\left.2 t|\nabla \phi| \cdot|\nabla| \nabla u\right|^{2} \mid \geq-\frac{2 t|\nabla \phi|^{2}|\nabla u|^{2}}{\varepsilon \phi}
$$

we get

$$
\begin{array}{r}
\left(L-\partial_{t}\right) G \geq-\frac{G}{t}+\frac{2(1-\varepsilon) \phi t}{m}\left(|\nabla u|^{2}-u_{t}\right)^{2}-2 K \phi t|\nabla u|^{2}-2|\nabla u| \cdot|\nabla G| \\
-2 t|\nabla u|^{3}|\nabla \phi|+t|\nabla u|^{2} L \phi-\frac{2 t|\nabla \phi|^{2}|\nabla u|^{2}}{\varepsilon \phi} .
\end{array}
$$

We assume that $\sup G>0$, otherwise the proof is done. Since $G(0, \cdot)=0$ and $\left.N G\right|_{\partial M} \geq 0$, we can apply Theorem 1.2. Let $\left\{\left(t_{n}, x_{n}\right)\right\}$ be fixed in Theorem 1.2 with, for example, $\varepsilon=1 / 2$. Then,

$$
\left(L-\partial_{t}\right) G\left(t_{n}, x_{n}\right) \leq \frac{G^{3 / 2}\left(t_{n}, x_{n}\right)}{n} \quad \text { and } \quad|\nabla G|\left(t_{n}, x_{n}\right) \leq \frac{G^{3 / 2}\left(t_{n}, x_{n}\right)}{n} .
$$

From now on, we evaluate functions at the point $\left(t_{n}, x_{n}\right)$, so that $t=t_{n}$.

Let $\mu=|\nabla u|^{2} / G$. We have

$$
|\nabla u|^{2}-u_{t}=\left(\mu-\frac{(\mu t-1) \phi}{\alpha t}\right) G=\frac{\mu t(\alpha-\phi)+\phi}{\alpha t} G .
$$

Combining this with (3-3) and (3-4), we arrive at

$$
\frac{2(1-\varepsilon) \phi(\mu t(\alpha-\phi)+\phi)^{2}}{m \alpha^{2} t} G^{2}
$$

$$
\leq \frac{G^{3 / 2}}{n}+\frac{G}{t}+\frac{2 \sqrt{\mu} G^{2}}{n}+2 t|\nabla \phi|(\mu G)^{3 / 2}+\left(2 k \phi+2 \varepsilon^{-1} \phi^{-1}|\nabla \phi|^{2}-L \phi\right) \mu t G .
$$

Since it is easy to see that

$$
(\mu t(\alpha-\phi)+\phi)^{2} \geq \max \left\{\phi^{2}, 4 \mu t(\alpha-\phi) \phi,(2 t(\alpha-\phi))^{3 / 2} \sqrt{\phi} \mu^{3 / 2}\right\},
$$


we may multiply both sides of (3-5) by $t(\mu t(\alpha-\phi)+\phi)^{-2} G^{-2}$ to obtain

$$
\begin{aligned}
\frac{2(1-\varepsilon) \phi}{m \alpha^{2}} & \leq \frac{c^{\prime} t}{n(1 \wedge \sqrt{G})}+\frac{1}{\phi^{2} G}+\frac{2 K+2 \varepsilon^{-1}|\nabla \log \phi|^{2}-\phi^{-1} L \phi}{4(\alpha-\phi) G} t \\
& \leq \frac{|\nabla \log \phi| \sqrt{t \phi}}{(\alpha-\phi)^{3 / 2} \sqrt{2 G}} \\
& +\frac{c^{\prime} t}{n(1 \wedge \sqrt{G})}+\frac{1}{\phi^{2} G}+\frac{2 K+2 \varepsilon^{-1}|\nabla \log \phi|^{2}-\phi^{-1} L \phi}{4(\alpha-\phi) G} \\
& +\frac{|\nabla \log \phi|^{2} m \alpha^{2}(1+\varepsilon) t}{16(\alpha)^{3} \varepsilon(1-\varepsilon) G}+\frac{2(1-\varepsilon) \varepsilon \phi}{m \alpha^{2}(1+\varepsilon)}
\end{aligned}
$$

for some constant $c^{\prime}>0$. Taking $n \rightarrow \infty$ and noting that $\phi \geq 1$, we conclude that $\theta:=\sup G$ satisfies

$$
\begin{aligned}
\frac{2(1-\varepsilon)}{m \alpha^{2}(1+\varepsilon)} \leq \frac{1}{\theta}\left(1+\frac{2 K+2 \varepsilon^{-1}\|\nabla \log \phi\|_{\infty}^{2}+\sup \left(-\phi^{-1} L \phi\right)}{4\left(\alpha-\|\phi\|_{\infty}\right)} T\right. & \\
& \left.+\frac{\|\nabla \log \phi\|_{\infty}^{2} m \alpha^{2}(1+\varepsilon) T}{16\left(\alpha-\|\phi\|_{\infty}\right)^{3} \varepsilon(1-\varepsilon)}\right) .
\end{aligned}
$$

Combining this with $\theta \geq G(T, x)=T\left(\phi(x)|\nabla u|^{2}(T, x)-\alpha u_{t}(T, x)\right)$ for $x \in M$, we obtain

$$
\begin{aligned}
\phi(x)|\nabla u|^{2} & (T, x)-\alpha u_{t}(T, x) \\
\leq \frac{m \alpha^{2}(1+\varepsilon)}{2(1-\varepsilon)}\left(\frac{1}{T}+\frac{2 K+2 \varepsilon^{-1}\|\nabla \log \phi\|_{\infty}^{2}+\sup \left(-\phi^{-1} L \phi\right)}{4\left(\alpha-\|\phi\|_{\infty}\right)}\right. & \left.\quad+\frac{\|\nabla \log \phi\|_{\infty}^{2} m \alpha^{2}(1+\varepsilon)}{16\left(\alpha-\|\phi\|_{\infty}\right)^{3} \varepsilon(1-\varepsilon)}\right)
\end{aligned}
$$

for all $x \in M$. Then the proof is completed since $T>0$ is arbitrary.

Proof of Corollary 1.4. By Theorem 1.3, the proof is standard according to [Li and Yau 1986]. For $x, y \in M$, let $\gamma:[0,1] \rightarrow M$ be the shortest curve in $M$ linking $x$ and $y$ such that $|\dot{\gamma}|=\rho(x, y)$. Then, for any $s, t>0$ and $f \in C_{b}^{\infty}(M)$, it follows from (1-5) that

$$
\begin{aligned}
\frac{\mathrm{d}}{\mathrm{d} r} \log P_{t+r s} f\left(\gamma_{r}\right) & =\left.s \partial_{u} \log P_{u} f\left(\gamma_{r}\right)\right|_{u=t+r s}+\left\langle\dot{\gamma}_{r}, \nabla P_{t+r s} f\left(\gamma_{r}\right)\right\rangle \\
\geq & \frac{s}{\alpha}\left|\nabla \log P_{t+r s} f\right|^{2}\left(\gamma_{r}\right)-\rho(x, y)|\nabla \log f|\left(\gamma_{r}\right) \\
& -s\left(\frac{m(1+\varepsilon) \alpha}{2(1-\varepsilon)(t+r s)}+\frac{m \alpha K(\phi, \varepsilon, \alpha)}{4\left(\alpha-1\|\phi\|_{\infty}\right)}\right) \\
& \geq-\frac{\alpha}{4 s}-s\left(\frac{m(1+\varepsilon) \alpha}{2(1-\varepsilon)(t+r s)}+\frac{m \alpha K(\phi, \varepsilon, \alpha)}{4\left(\alpha-\|\phi\|_{\infty}\right)}\right) .
\end{aligned}
$$


We complete the proof by integrating with respect to $\mathrm{d} r$ over $[0,1]$.

\section{Proof of Theorem 1.6}

We first provide a simple proof of (1-9) under an extra assumption that $\left|\nabla P_{(\cdot)} f\right|$ is bounded on $[0, T] \times M$ for any $T>0$; we then drop this assumption by an approximation argument.

Lemma 4.1. If that $f \in C_{b}^{1}(M)$ is such that $\left|\nabla P_{(\cdot)} f\right|$ is bounded on $[0, T] \times M$ for any $T>0$, then (1-9) holds.

Proof. For any $\varepsilon>0$, let $\eta_{s}=\sqrt{\varepsilon+\left|\nabla P_{t-s} f\right|^{2}}\left(X_{s}\right)$ for $s \leq t$. By the Itô formula, we have

$$
\begin{aligned}
\mathrm{d} \eta_{s}=\mathrm{d} M_{s}+\frac{L\left|\nabla P_{t-s} f\right|^{2}-2\left\langle\nabla L P_{t-s} f, \nabla P_{t-s} f\right\rangle}{2 \sqrt{\left.\varepsilon+\left|\nabla P_{t-s} f\right|^{2}\right)^{2}}}\left(X_{s}\right) \mathrm{d} s \\
\quad-\frac{\left.\left.|\nabla| \nabla P_{t-s} f\right|^{2}\right|^{2}}{4\left(\varepsilon+\left|\nabla P_{t-s} f\right|^{2}\right)^{3 / 2}}\left(X_{s}\right) \mathrm{d} s+\frac{N\left|\nabla P_{t-s} f\right|^{2}}{2 \sqrt{\varepsilon+\left|\nabla P_{t-s} f\right|^{2}}}\left(X_{s}\right) \mathrm{d} l_{s}
\end{aligned}
$$

for $s \leq t$, where $M_{s}$ is a local martingale. Combining this with (1-8) and (3-1), with $\kappa_{1}$ in place of $K_{0}$, we obtain

$$
\begin{aligned}
\mathrm{d} \eta_{s} & \geq \mathrm{d} M_{s}-\frac{\kappa_{1}\left|\nabla P_{t-s} f\right|^{2}}{\sqrt{\varepsilon+\left|\nabla P_{t-s} f\right|^{2}}}\left(X_{s}\right) \mathrm{d} s-\frac{\kappa_{2}\left|\nabla P_{t-s} f\right|^{2}}{\sqrt{\varepsilon+\left|\nabla P_{t-s} f\right|^{2}}}\left(X_{s}\right) \mathrm{d} l_{s} \\
& \geq \mathrm{d} M_{s}-\kappa_{1}\left(X_{s}\right) \eta_{s} \mathrm{~d} s-\kappa_{2}\left(X_{s}\right) \eta_{s} \mathrm{~d} l_{s} \quad \text { for } s \leq t .
\end{aligned}
$$

Now $\eta_{s}$ is bounded on $[0, t]$, and by the proof of [Wang 2005b, Lemma 2.1] we have $\mathrm{Ee}^{\lambda l_{t}}<\infty$ for all $\lambda>0$. This implies that

$$
[0, t] \ni s \mapsto \sqrt{\varepsilon+\left|\nabla P_{t-s} f\right|^{2}\left(X_{s}\right)} \exp \left(\int_{0}^{s} \kappa_{1}\left(X_{s}\right) \mathrm{d} s+\int_{0}^{s} \kappa_{2}\left(X_{s}\right) \mathrm{d} l_{s}\right)
$$

is a submartingale for any $\varepsilon>0$. Letting $\varepsilon \downarrow 0$ we conclude that

$$
[0, t] \ni s \mapsto\left|\nabla P_{t-s} f\right|\left(X_{s}\right) \exp \left(\int_{0}^{s} \kappa_{1}\left(X_{s}\right) \mathrm{d} s+\int_{0}^{s} \kappa_{2}\left(X_{s}\right) \mathrm{d} l_{s}\right)
$$

is a submartingale as well.

According to Lemma 4.1, it suffices to confirm the boundedness of $\left|\nabla P_{(\cdot)} f\right|$ on $[0, T] \times M$ for any $T>0$ and $f \in C_{b}^{1}(M)$. We shall start from $f \in C_{0}^{\infty}(M)$ with $\left.N f\right|_{\partial M}=0$, then pass to $f \in C_{b}^{1}(M)$ by combining an approximation argument and Lemma 4.1.

Case a. Let $f \in C_{0}^{\infty}(M)$ with $\left.N f\right|_{\partial M}=0$. We have

$$
P_{t} f=f+\int_{0}^{t} P_{s} L f \mathrm{~d} s .
$$


Since $L f$ is bounded, there is a $c>0$ such that $L f+c \geq 1$. Applying Corollary 1.5 with for example $\alpha=2+\sigma d r_{0}$ and $\varepsilon=1 / 2$, but using $L f+c$ in place of $f$, we obtain

$$
\begin{aligned}
\left|\nabla P_{s} L f\right| & =\left|\nabla P_{s}(L f+c)\right| \\
& \leq\|L f+c\|_{\infty}\left(\alpha\left\|P_{s} L^{2} f\right\|_{\infty}+\frac{m(1+\varepsilon) \alpha^{2}}{2(1-\varepsilon) s}+\frac{m \alpha^{2} K_{\varepsilon}}{4\left(\alpha-1-\sigma d r_{0}\right)}\right)^{1 / 2} \\
& \leq c_{1} / \sqrt{s} \quad \text { for } s \leq T
\end{aligned}
$$

for some constant $c_{1}>0$. Combining this with (4-1) we conclude that, for some constant $c_{2}>0$,

$$
\left|\nabla P_{t} f\right| \leq|\nabla f|+\int_{0}^{t} \frac{c_{1}}{\sqrt{s}} \mathrm{~d} s \leq c_{2} \quad \text { for } t \leq T .
$$

Case b. Let $f \in C_{0}^{\infty}(M)$. There exists a sequence of functions $\left\{f_{n}\right\}_{n \geq 1} \subset C_{0}^{\infty}(M)$ such that $\left.N f_{n}\right|_{\partial M}=0, f_{n} \rightarrow f$ uniformly as $n \rightarrow \infty$, and $\left\|\nabla f_{n}\right\|_{\infty} \leq 1+\|\nabla f\|_{\infty}$ holds for any $n \geq 1$; see for example [Wang 1994]. By Case a and Lemma 4.1, (1-9) holds with $f_{n}$ in place of $f$, so that

$$
\frac{\left|P_{t} f_{n}(x)-P_{t} f_{n}(y)\right|}{\rho(x, y)} \leq C \quad \text { for } t \leq T, n \geq 1, x \neq y
$$

for some constant $C>0$. Letting first $n \rightarrow 0$ and then $y \rightarrow x$, we conclude that $\left|\nabla P_{(\cdot)} f\right|$ is bounded on $[0, T] \times M$.

Case c. Let $f \in C_{b}^{\infty}(M)$. Let $\left.\left\{g_{n}\right\}_{n \geq 1} \subset C_{0}^{\infty}\right)$ be such that $0 \leq g_{n} \leq 1,\left|\nabla g_{n}\right| \leq 2$ and $g_{n} \uparrow 1$ as $n \uparrow \infty$. By Case b and Lemma 4.1, we may apply (1-9) to $g_{n} f$ in place of $f$ such that

$$
\frac{\left|P_{t}\left(g_{n} f\right)(x)-P_{t}\left(g_{n} f\right)(y)\right|}{\rho(x, y)} \leq C \text { for } t \leq T, n \geq 1, x \neq y
$$

for some constant $C>0$. By the same reason as in Case b, we conclude that $\left|\nabla P_{(\cdot)} f\right|$ is bounded on $[0, T] \times M$.

Case d. Finally, for $f \in C_{b}^{1}(M)$, there exist $\left\{f_{n}\right\}_{n \geq 1} \subset C_{b}^{\infty}(M)$ such that $f_{n} \rightarrow f$ uniformly as $n \rightarrow \infty$ and $\left\|\nabla f_{n}\right\|_{\infty} \leq\|\nabla f\|_{\infty}+1$ for any $n \geq 1$. The proof is completed by the same reason as in Cases b and c.

\section{Acknowledgment}

The author thanks the referee for useful comments and a number of corrections.

\section{References}

[Bakry and Émery 1984] D. Bakry and M. Émery, "Hypercontractivité de semi-groupes de diffusion”, C. R. Acad. Sci. Paris Sér. I Math. 299:15 (1984), 775-778. MR 86f:60097 Zbl 0563.60068 
[Bakry and Qian 1999] D. Bakry and Z. M. Qian, "Harnack inequalities on a manifold with positive or negative Ricci curvature", Rev. Mat. Iberoamericana 15:1 (1999), 143-179. MR 2000f:58052 Zbl 0924.58096

[Davies 1989] E. B. Davies, Heat kernels and spectral theory, Cambridge Tracts in Mathematics 92, Cambridge University Press, 1989. MR 90e:35123 Zbl 0699.35006

[Hsu 2002] E. P. Hsu, "Multiplicative functional for the heat equation on manifolds with boundary", Michigan Math. J. 50:2 (2002), 351-367. MR 2003f:58067 Zbl 1037.58024

[Kasue 1984] A. Kasue, "Applications of Laplacian and Hessian comparison theorems", pp. 333386 in Geometry of geodesics and related topics (Tokyo, 1982), edited by K. Shiohama, Adv. Stud. Pure Math. 3, North-Holland, Amsterdam, 1984. MR 86j:53062 Zbl 0578.53029

[Kendall 1987] W. S. Kendall, "The radial part of Brownian motion on a manifold: a semimartingale property”, Ann. Probab. 15:4 (1987), 1491-1500. MR 88k:60151 Zbl 0647.60086

[Ledoux 2000] M. Ledoux, "The geometry of Markov diffusion generators", Ann. Fac. Sci. Toulouse Math. (6) 9:2 (2000), 305-366. MR 2002a:58045 Zbl 0980.60097

[Li and Yau 1986] P. Li and S.-T. Yau, "On the parabolic kernel of the Schrödinger operator", Acta Math. 156:3-4 (1986), 153-201. MR 87f:58156 Zbl 0611.58045

[Qian 1998] Z. Qian, “A comparison theorem for an elliptic operator”, Potential Anal. 8:2 (1998), 137-142. MR 99d:58161 Zbl 0930.58012

[Wang 1994] F. Y. Wang, "Application of coupling methods to the Neumann eigenvalue problem", Probab. Theory Related Fields 98:3 (1994), 299-306. MR 94k:58153 Zbl 0791.58113

[Wang 1997] J. Wang, “Global heat kernel estimates”, Pacific J. Math. 178:2 (1997), 377-398. MR 98g:58168 Zbl 0882.35018

[Wang 2005a] F.-Y. Wang, Functional inequalities, Markov semigroups, and spectral theory, Elsevier, Amsterdam, 2005.

[Wang 2005b] F.-Y. Wang, "Gradient estimates and the first Neumann eigenvalue on manifolds with boundary”, Stochastic Process. Appl. 115:9 (2005), 1475-1486. MR 2006g:58054 Zbl 1088.58015

[Wang 2007] F.-Y. Wang, "Estimates of the first Neumann eigenvalue and the log-Sobolev constant on non-convex manifolds", Math. Nachr. 280:12 (2007), 1431-1439. MR 2008m:58050 Zbl 1130.58019

[Yau 1975] S. T. Yau, "Harmonic functions on complete Riemannian manifolds", Comm. Pure Appl. Math. 28 (1975), 201-228. MR 55 \#4042 Zbl 0291.31002

Received November 13, 2008. Revised August 25, 2009.

FENG-YU WANG

SCHOOL of MATHEMATICAL SCIENCES

BEIJING NORMAL UNIVERSITY

BEIJING 100875

CHINA

wangfy@bnu.edu.cn

and

Mathematics Department

SWANSEA UNIVERSITY

SINGLETON PARK SA2 8PP

UNITED KINGDOM

F.Y.Wang@swansea.ac.uk 